\title{
Oral Anticoagulation Timing in Patients with Acute Ischemic Stroke and Atrial Fibrillation
}

\author{
Po-Yin Chang ${ }^{1, *}$ Wei-Ting Wang ${ }^{2,3,4,5, *(0)}$ Wei-Lun Wu ${ }^{6}$ Hui-Chin Chang ${ }^{6}$ Chen-Huan Chen ${ }^{4,7,8}$ \\ Yi-Wen Tsai ${ }^{6, *}$ Shih-Hwa Chiou ${ }^{3,4,5}$ Gregory Y. H. Lip ${ }^{9,10}$ Hao-Min Cheng ${ }^{2,3,7,8,11, * \mathbb{C}}$ \\ Chern-En Chiang 2,12
}

${ }^{1}$ Office of Surveillance and Epidemiology, Center for Drug Evaluation and Research, U.S. Food and Drug Administration, Maryland, United States

${ }^{2}$ Division of Cardiology, Department of Medicine, Taipei Veterans General Hospital, Taipei City, Taiwan

${ }^{3}$ Department of Medicine, National Yang Ming Chiao Tung University, Taipei, Taiwan

${ }^{4}$ School of Medicine, National Yang Ming Chiao Tung University, Taipei, Taiwan

${ }^{5}$ Institute of Clinical Medicine, National Yang Ming Chiao Tung University, Taipei, Taiwan

6 Institute of Health and Welfare Policy, National Yang Ming Chiao Tung University, Taipei, Taiwan

${ }^{7}$ Department of Medical Education, Taipei Veterans General Hospital, Taipei, Taiwan

Thromb Haemost 2022;122:939-950.
Address for correspondence Hao-Min Cheng, MD, PhD, No. 201, Section 2, Shih-Pai Road, Beitou District, Taipei 11217, Taiwan (e-mail: hmcheng@vghtpe.gov.tw).

Yi-Wen Tsai, PhD, No. 155, Section 2, Li-Nong St, Beitou District, Taipei 11221, Taiwan (e-mail: ywtsai@nycu.edu.tw).

8 Institute of Public Health, National Yang Ming Chiao Tung University, Taipei, Taiwan

${ }^{9}$ Liverpool Centre for Cardiovascular Science, University of Liverpool and Liverpool Heart and Chest Hospital, Liverpool, United Kingdom

${ }^{10}$ Aalborg Thrombosis Research Unit, Department of Clinical Medicine, Aalborg University, Aalborg, Denmark

${ }^{11}$ Center for Evidence-based Medicine, Taipei Veterans General Hospital, Taipei, Taiwan

${ }^{12}$ General Clinical Research Center, Taipei Veterans General Hospital, Taipei, Taiwan
Abstract
Keywords
- atrial fibrillation
- acute ischemic stroke
- oral anticoagulants
- timing
- stroke recurrence

Background and Purpose Oral anticoagulants (OACs) prevent stroke recurrence and vascular embolism in patients with acute ischemic stroke (AIS) and atrial fibrillation (AF). Based on empirical consensus, current guidance recommends a "1-3-6-12 days" rule to resume OACs after AIS. This study investigated the suitability of guidelinerecommended timing for $\mathrm{OAC}$ initiation.

Methods Using data of 12,307 AF patients hospitalized for AIS, for the period 2012 to 2016, in Taiwan's National Health Insurance Research Database, we constructed a sequence of cohorts of OAC users and propensity score-matched nonusers, creating one cohort on each day of OAC initiation for 30 days since admission. Composite outcome included effectiveness (cardiovascular death, ischemic stroke, myocardial infarction, transient ischemic attack, systemic embolism, and venous thromboembolism) and safety (intracranial hemorrhage, gastrointestinal bleeding, and hematuria) outcomes. Comparing with nonusers, we examined the risks in the early OAC use

\footnotetext{
These authors contributed equally to this work.

Note: The review process for this paper was fully handled by

Christian Weber, Editor-in-Chief.
}

received

June 2, 2021

accepted after revision

October 11, 2021

published online

October 14, 2021
DOI https://doi.org/

$10.1055 / \mathrm{a}-1669-4987$

ISSN 0340-6245. (c) 2021. The Author(s).

This is an open access article published by Thieme under the terms of the Creative Commons Attribution License, permitting unrestricted use, distribution, and reproduction so long as the original work is properly cited. (https://creativecommons.org/licenses/by/4.0/)

Georg Thieme Verlag KG, Rüdigerstraße 14, 70469 Stuttgart, Germany 
(within 1-3-6-12 days) or guideline-recommended delayed use. Indirect comparison between the early and delayed use was conducted using mixed treatment comparison. Results Across the AIS severity, the risks of composite or effectiveness outcome were lower in OAC users than nonusers, and the risks were similar between the early and delayed use groups. In patients with severe AIS, early OAC use was associated with an increased risk of safety outcome, with a hazard ratio (HR) of 1.67 (confidence interval $[\mathrm{Cl}]: 1 \cdot 30-2 \cdot 13)$ compared with nonusers and a HR of $1.44(\mathrm{Cl}: 0.99-2 \cdot 09)$ compared with the delayed use.

Conclusion Our study findings support an early OAC initiation in AF patients with mild-to-moderate AIS and a routine delayed use of OACs can be considered in those with severe AIS to avoid a serious bleeding event.

\section{Introduction}

Stroke is a leading cause of mortality and disability, resulting in substantial economic costs in terms of poststroke care. ${ }^{1}$ Cardioembolic strokes, most frequently caused by atrial fibrillation (AF), ${ }^{2}$ are found to be related to worse outcomes compared with other non-AF-related strokes.

Lifelong use of oral anticoagulants (OACs) has been recommended for secondary stroke prevention. ${ }^{3,4}$ However, the optimal timing to resume OAC in AF patients with acute ischemic stroke (AIS) remains a clinical challenge. Early non-vitamin $\mathrm{K}$ antagonist OACs (NOACs) within 2 days of AIS had been shown to be associated with a $5 \%$ rate of hemorrhagic transformation, ${ }^{5}$ whereas a delayed initiation may leave the patients at an increased risk of recurrent ischemic stroke. The 2018 European Heart Rhythm Association practical guide proposed a "1-3-6-12 days rule" to resume OAC after an AIS in patients with $\mathrm{AF}^{4,6}$ based on expert consensus opinion without supporting evidence from large-scale randomized controlled trials (RCTs). Recently, one meta-analysis of individual-level data from seven prospective observational studies, including CROMIS-2, ${ }^{7}$ RAF, ${ }^{8}$ RAF-NOACs, ${ }^{9}$ SAMURAI, ${ }^{10}$ NOACISP, ${ }^{11}$ Erlangen, ${ }^{12}$ and Verona ${ }^{13}$ registry, suggested that that early NOAC treatment after AIS, when compared with vitamin $\mathrm{K}$ antagonist (VKA), was associated with a reduced risk of intracranial hemorrhage (ICH). ${ }^{14}$ Interestingly, Mizoguchi and colleagues compared the early ( $\leq 3$ days) with the delayed ( $\geq 4$ days) initiation of NOACs after AIS or transient ischemic attack (TIA) in the SAMURAI study and did not observe a difference in risks of stroke, major bleeding, and death between the groups. ${ }^{15}$ The study by Mizoguchi and coauthors, however, did not address the potential immortal-time bias because patients who initiated NOACs in the delayed period, by definition, had to be alive and free of ischemic stroke in the early period (and thus to be "immortal" to outcomes of interest.)

Four ongoing RCTs, including ELAN (NCT03148457, Switzerland), OPTIMAS (EudraCT, 2018003859-38, United Kingdom), TIMING (NCT02961348, Sweden), and START (NCT03021928, United States), are to determine the optimal time for initiating OACs after AIS. However, these RCTs only compare the early and delayed initiation of NOACs with fixed intervals, without stratified randomization based on prespecified AIS severity, except ELAN stratifies patients based on the size of the infarction. Moreover, these RCTs fail to investigate the comparative effectiveness and safety of VKA in patients with AIS secondary to AF.

The present study aimed to examine the benefit and risk of early and delayed use of OACs, including NOACs and VKAs, in AF patients hospitalized for AIS. Immortal-time bias is a challenging issue in comparing different strategies of treatment initiation in observational studies, and we constructed a sequence of stroke severity-specific cohorts with propensity score (PS) matching to reduce immortal-time bias and confounding bias. The study results could provide real-world evidence of the optimal timing to initiate OACs after an AIS event among patients with AF.

\section{Methods}

Taiwan National Health Insurance Research Databases Taiwan initiated its single-payer, universal National Health Insurance program in March 1995. Enrolment is mandatory. As of 2020, membership consisted of approximately $23,622,000$ individuals (99.9\% of Taiwan's population). The National Health Insurance Research Database (NHIRD) captures all medical claims, including disease diagnoses, procedures, and prescription fills in the records of inpatient, outpatient, and emergency visits since 2000 for research purposes. The consistency, reliability, and disease diagnostic accuracy of the NHIRD for research in cardiovascular, bleeding, and mortality outcomes among patients with $\mathrm{AF}$ and/or AIS have been validated. ${ }^{16-23}$ The Institutional Review Board of the National Yang-Ming University, Taiwan, approved this research study (YM104104E).

\section{Study Population}

The base cohort included 268,715 patients who presented a new AIS ("index stroke event") from January 1, 2012 to December 31, 2016, who did not have a diagnosis of hemorrhagic stroke or TIA on the admission day, and who did not have any inpatient diagnosis of ischemic stroke within 5 years before the index stroke event (-Fig. 1). Using 


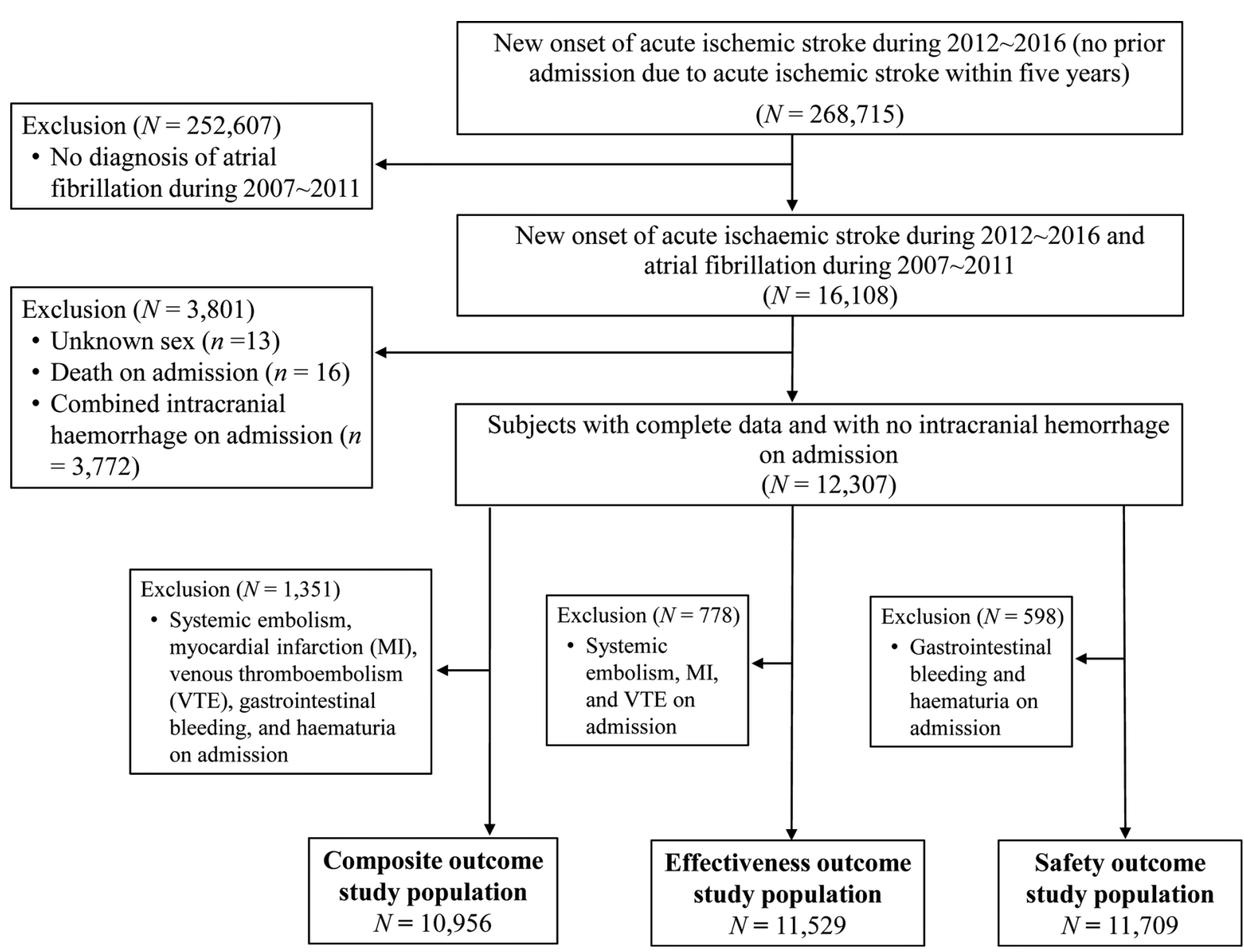

Fig. 1 Flowchart of patient selection. AIS, acute ischemic stroke.

algorithms validated in the NHIRD, AIS was validated by the noncontrast computed tomography (CT) or magnetic resonance imaging (MRI), ${ }^{21}$ and AF was defined as having at least one inpatient or outpatient record of the International Classification of Diseases (ICD)-9 or ICD-10 diagnosis code for AF as the primary diagnosis, or having at least two records of AF diagnosis as the secondary diagnosis within 5 years before the index stroke event ${ }^{23,24}$ (-Supplementary Table S1, available in the online version). The final study population consisted of 12,307 AF patients with a new AIS, after excluding patients who lacked AF diagnosis in 2007 to 2011 ( $n=252,607)$, were of unknown sex $(n=13)$, died on admission $(n=16)$, or had an ICH diagnosis on admission $(n=3,772)$.

\section{Severity of Acute Ischemic Stroke}

We calculated a validated stroke severity index (SSI) to categorize the index stroke event into mild (SSI $\leq 5$ ), moderate $(5<$ SSI $\leq 12)$, and severe $($ SSI $>12)$ stroke. $^{25-27}$ Stroke severity assessed by the National Institutes of Health Stroke Scale (NIHSS) cannot be captured in the administrative claims data, and SSI was closely correlated with NIHSS ${ }^{27}$ and performed well in 30-day and 1-year mortality prediction in validation studies. ${ }^{26}$ SSI calculation was based on the number of the following tests on admission: airway suctioning, bacterial sensitivity test, general ward stay, intensive care unit stay, nasogastric intubation, osmotherapy (mannitol or glycerol), and urinary catheterization. ${ }^{27}$

\section{Guideline-Recommended OAC Use in AF Patients Hospitalized for Stroke}

Guidelines recommend initiation of OACs, including NOACs or warfarin, on the 4th day of admission for a minor AIS, 7th day for a moderate AIS, and 13th day for a severe AIS. ${ }^{4,6}$ Depending on the stroke severity, "early use" referred to initiation of OACs within 3 (minor AIS), 6 (moderate AIS), or 12 days (severe AIS) of admission; "delayed use" referred to initiation of OACs between the guideline-recommended initiation day and the 30th day of admission. On a specific day, the exposed group included patients who initiated OACs and the unexposed group included patients who did not initiate OACs. Information of NOAC or warfarin initiation was based on prescriptions in inpatient and outpatient settings. Within 30 days of admission, patients who had prescriptions of NOAC only or VKA only were categorized into the "NOAC group" or the "VKA group," respectively, and patients who had prescriptions of both NOAC and VKA were categorized into the "both group."

\section{Composite Outcome of Effectiveness and Safety}

The primary outcome was the first occurrence of a composite outcome of an effectiveness or a safety event. Effectiveness 
outcomes included ischemic stroke, myocardial infarction (MI), TIA, systemic embolism, venous thromboembolism (VTE), and cardiovascular death. Safety outcomes included $\mathrm{ICH}$, gastrointestinal (GI) bleeding, and hematuria. These outcome events were identified using ICD-9 or ICD-10 diagnosis codes in inpatient records based on validated algorithms in the NHIRD ${ }^{16,18-23,28}$ (-Supplementary Table S1, available in the online version).

\section{Immortal-Time Bias and the Sequence of Cohorts with PS-Matching on Each Day of OAC Initiation}

Immortal time refers to a period of cohort follow-up when study subjects cannot have outcome(s) because of exposure definition. For example, patients who initiated an OAC on the sixth day of admission had to be alive and cannot develop any outcome from the first to the sixth day. Additionally, patients may initiate OACs on a specific day based on physicians' decisions, patients' clinical status, and possibly guideline suggestions. ${ }^{6}$ Consequently, the early and delayed use groups likely have different baseline risks of outcomes, and a direct comparison between the two groups could introduce immortal-time bias and confounding bias. ${ }^{29,30}$ To reduce these biases, we constructed a sequence of PS-matched cohorts of OAC users and nonusers, creating one cohort on each day of OAC initiation for 30 days since admission (-Fig. 2) ${ }^{30}$ The day of OAC initiation was defined as the index date for each cohort. Across the three categories of stroke severity and the
30 possible days of OAC initiation, we constructed 90 PSmatched cohorts nested within the study population ( $n=12,307$ ) for each of the composite outcome, effectiveness outcome, and safety outcome. The analytic sample of AF patients eligible for PS-matching included 10,956 patients for the composite outcomes, 11,529 patients for the effectiveness outcomes, and 11,709 patients for the safety outcomes (-Fig. 1).

Follow-up for each PS-matched cohort started from the cohort index date until the first occurrence of a composite outcome event, noncardiovascular death, loss to follow-up, initiation of OACs in an unexposed group, or December 31, 2017.

\section{Statistical Analyses}

On each of the cohort index dates, we calculated PS using a logistic regression model that included age, sex, use of medication (antihypertensive drugs, antidiabetic drugs, lipid-lowering drugs, OACs, antiplatelets, nonsteroid anti-inflammatory drugs), and medical history of liver disease, peptic ulcer, hypertension, dyslipidemia, ischemic heart disease, ICH, TIA, alcohol intoxication, GI bleeding, hematuria, VTE, systemic embolism, congestive heart failure (CHF), $\mathrm{MI}$, peripheral vascular disease (PVD), cerebrovascular accident, diabetes mellitus, and chronic kidney disease (CKD). We 1:1 matched OAC users to nonusers using the greedy nearest-neighbor technique within a specified caliper width

Use of OACs in AF patients hospitalized for mild AIS

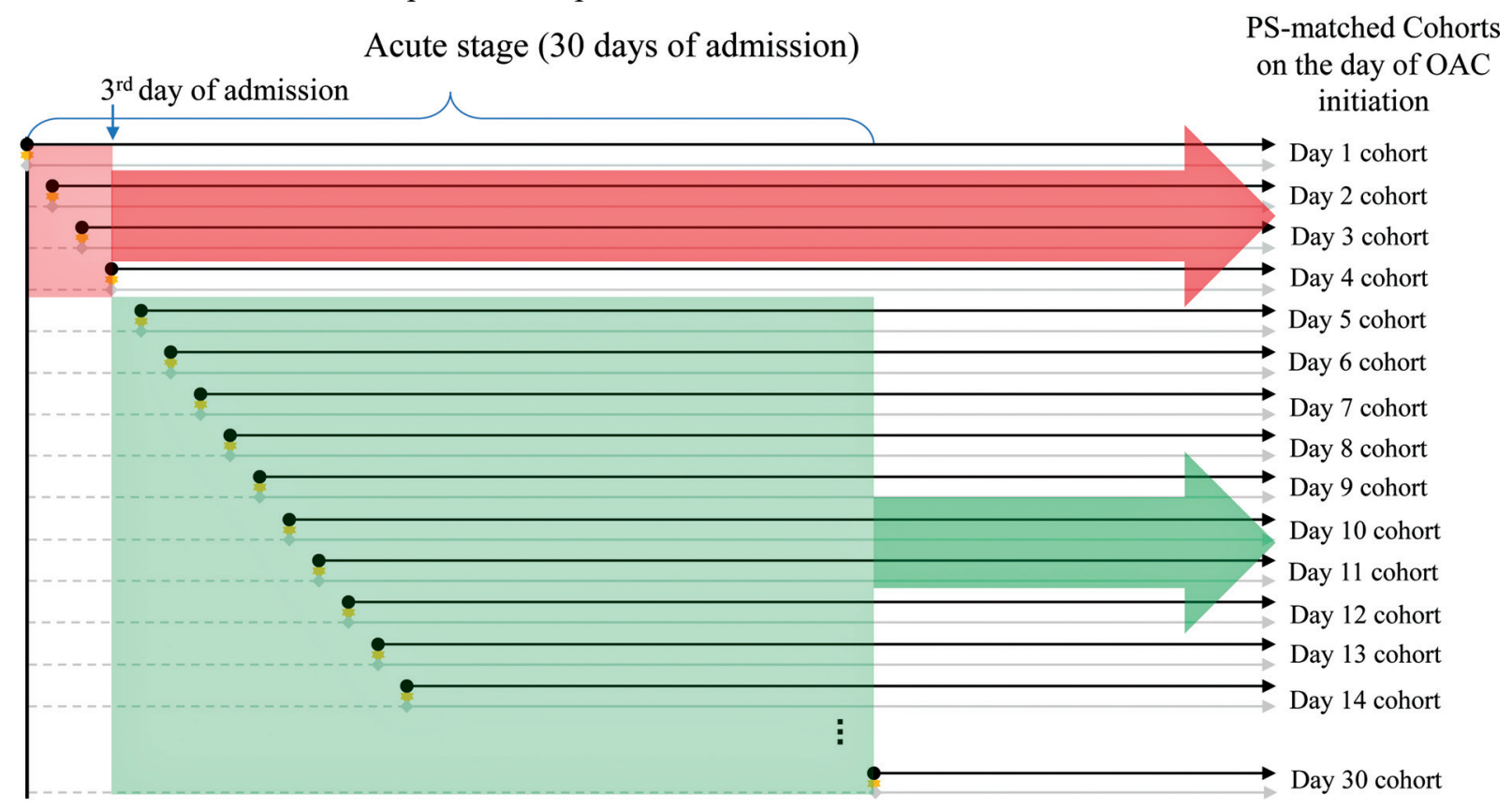

- OACs initiation date (index date)

Index date for no use group

Early OAC use
Delayed OAC use

- - - Immortal time between the AIS admission day and index date

1:1 propensity score (PS) match between OAC use and no use groups

Early use and no use comparison
Delayed use and no use comparison

Fig. 2 A sequence of propensity-score matched cohorts on each day of OAC initiation from the first to the 30th day of admission, using AF patients with mild AIS as an example. AF, atrial fibrillation; AIS, acute ischemic stroke; OAC, oral anticoagulant. 
of 0.25 of the standard deviation of the logit of the PS. ${ }^{31} \mathrm{~A}$ nonuser was allowed to be matched to multiple OAC users who initiated OACs on different days. Nonusers could later initiate OACs and become OAC users.

For each level of AIS severity, we pooled all PS-matched cohorts into one analytic sample. ${ }^{32}$ Using nonusers as the reference, we performed Cox proportional-hazards models to estimate hazard ratios (HRs) and 95\% confidence intervals (CIs) for outcomes in the early use and separately in the delayed use. We stratified Cox models on the index date and adjusted for the PS-matching variables. We used a robust variance estimate to account for within-person correlation. ${ }^{32}$

We used the cumulative incidence function ${ }^{33}$ to calculate cumulative incidence to account for possible competing risks. ${ }^{34}$ Categorical variables were expressed as the number (percentage) and assessed using the Chi-square $\left(\mathrm{X}^{2}\right)$ or Fisher's exact test.

\section{Mixed Treatment Comparison}

We indirectly compared the risk of outcomes in the early with the delayed use groups using a random-effects model. ${ }^{35}$ The indirect comparisons were based on the assumptions of cohort independence and consistency between direct and indirect comparisons. We applied the results comparing the exposed with the unexposed group as direct evidence, and extrapolated the indirect comparison from the direct evidence.

\section{Net Clinical Benefit Analysis}

We performed a net clinical benefit (NCB) analysis, proposed by Singer et $\mathrm{al}^{36}$, to examine the risk and benefit profile of early or delayed OAC use, compared with the no use group. The NCB was calculated as: (rate of effectiveness outcome in the no use group - rate of effectiveness outcome in the early [or delayed] use group) - weighting factor $\times$ (rate of safety outcome in the early [or delayed] use group - rate of safety outcome in the no use group). The $95 \%$ CIs were calculated from rate differences and standard errors estimates using Poisson regression. The weighting factor reflects the relative impact of a safety outcome while receiving an early or a delayed OAC, as opposed to experiencing an effectiveness outcome while not using OACs. We selected three weighting factors $(1.5,2.0$, and 3.0) based on publications of the risk and benefit of warfarin use in AF patients. ${ }^{36,37}$

\section{Results}

Of the 10,956 patients with $\mathrm{AF}$ in the composite outcome analysis (-Supplementary Table S2, available in the online version), $41 \cdot 2 \%(n=4,513)$ had a mild stroke, $23.6 \%$ $(n=2,582)$ had a moderate stroke, and $35 \cdot 2 \%(n=3,861)$ had a severe stroke. Among patients with AF, the proportion of guideline-recommended use of OACs decreased with an increasing stroke severity (early use: $29 \cdot 0,26 \cdot 8$, and $21.8 \%$; delayed use: $25 \cdot 9,22 \cdot 2$, and $14.5 \%$ in mild, moderate, and severe stroke, respectively). Conversely, the proportion of patients with no OAC use increased $(45 \cdot 1,51 \cdot 0$, and $63.7 \%$ in mild, moderate, and severe stroke, respectively).
Across the stroke severity, AF patients who did not initiate OACs after an AIS tended to be older and have more comorbidities than those who did (-Table 1, - Supplementary Tables S3-S13, available in the online version). For example, peptic ulcers, hypertension, ICH, GI bleeding, CHF, MI, PVD, diabetes, and CKD were more common in AF patients with mild stroke in the no use group than those in the early or delayed OAC use group (all $p$-values before PS-matching: $<0.05$; - Table 1). Patients in the no use group were more likely to use antiplatelet therapy and less likely to use OAC at baseline than those in the OAC use groups ( $p$-values before PS-matching: <0.05; - Table 1). After PS-matching, differences between the OAC use and no use groups disappeared.

As the stroke severity increased from mild to severe (-Supplementary Table S14, available in the online version), the incidence of composite outcome increased (444.5 to 928.3 cases per 1,000 person-years), as did the incidence of effectiveness outcome (292.1 to 654.7 cases per 1,000 person-years). The incidence of safety outcome did not vary substantially (149.5 to 196.4 cases per 1,000 person-years).

\section{Use of OACs versus No Use and the Risk of Outcomes}

AF Patients with Mild or Moderate Stroke

When compared with no OAC use, the early or delayed use was associated with a decreased risk of composite outcome with HR ranging from 0.73 (95\% CI: 0.62-0.85) to 0.82 (95\% CI: 0.67-1.00). The OAC use was not associated with an increased risk of safety outcomes (-Table $\mathbf{2}$ ).

\section{AF Patients with Severe Stroke}

Early use of OACs, compared with no use, was associated with a 0.79 -fold (95\% CI: $0.68-0.92$ ) risk of composite outcome, 0.82-fold (95\% CI: 0.70-0.95) risk of effectiveness outcome, and 1.67 -fold (95\% CI: 1.30-2.13) risk of safety outcomes. In NOAC- and warfarin-specific analyses, early use of NOAC was associated with a 0.58 -fold (95\% CI: 0.39-0.86) risk of effectiveness outcome and a 2.10 -fold (95\% CI: 1.13-3.92) risk of safety outcome. On the contrary, delayed use of warfarin was associated with a 0.52-fold (95\% CI: 0.35-0.71) risk of effectiveness outcomes and was not associated with an increased risk of safety outcomes (- Table $\mathbf{2}$ ).

\section{Early versus Delayed Use of OACs and the Risk of Outcomes}

Across the stroke severity level, the risk of composite or effectiveness outcomes did not significantly differ between the early use and the delayed use groups (-Table 3 ). However, a trend of an increased risk of safety outcomes associated with the early use of OACs was observed, particularly in patients with severe stroke (HR: 1.44, 95\% CI: 0.992.09, delayed use as reference). In AF patients with severe stroke, the risk of effectiveness outcome was lower in the early use than the delayed use of NOAC (HR: 0.57, 95\% CI: $0.35-0.91$ ); the opposite was observed in comparing the early use with the delayed use of warfarin (HR: $1.88,95 \% \mathrm{CI}$ : 1.33-2.68). 


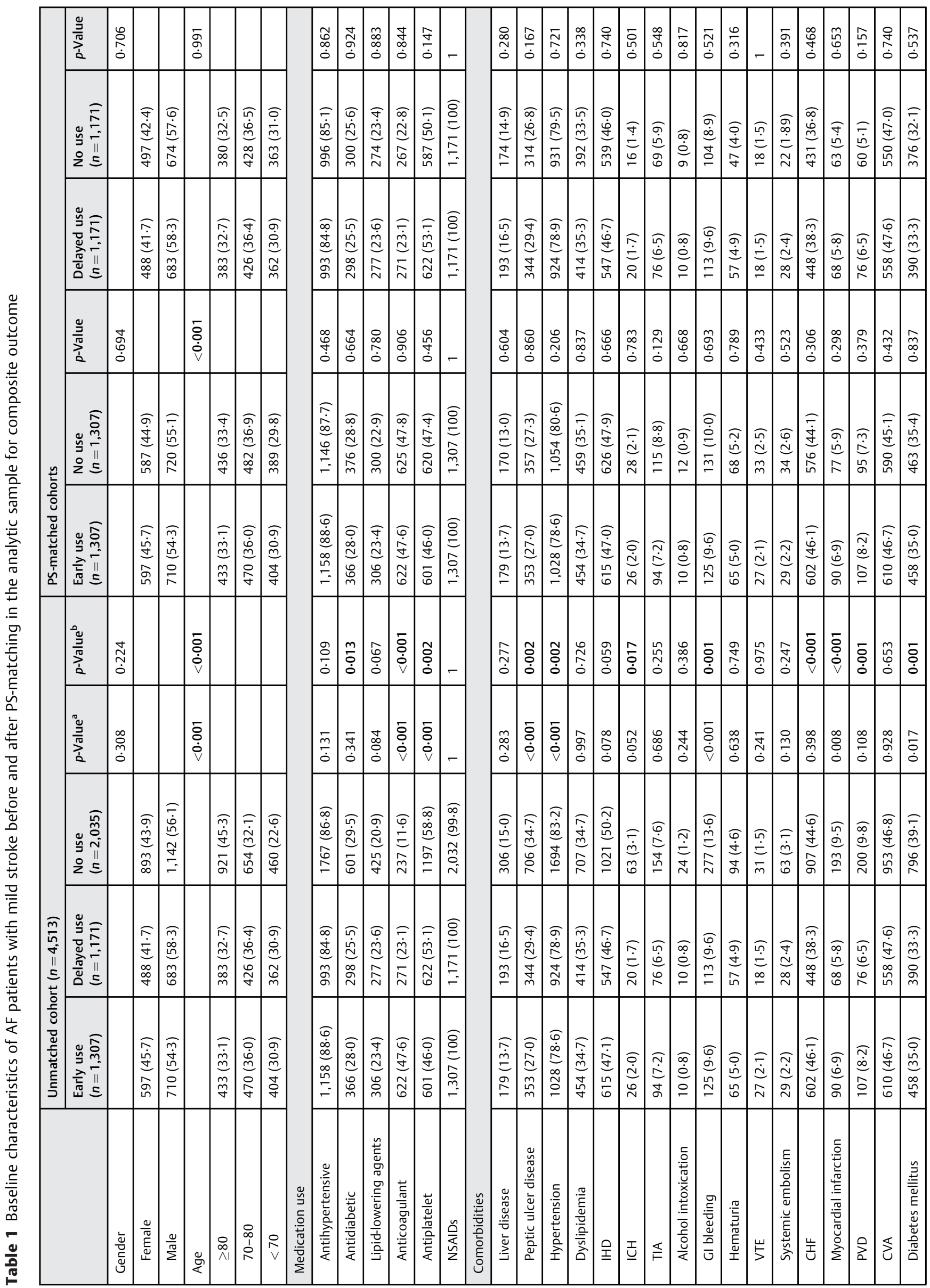




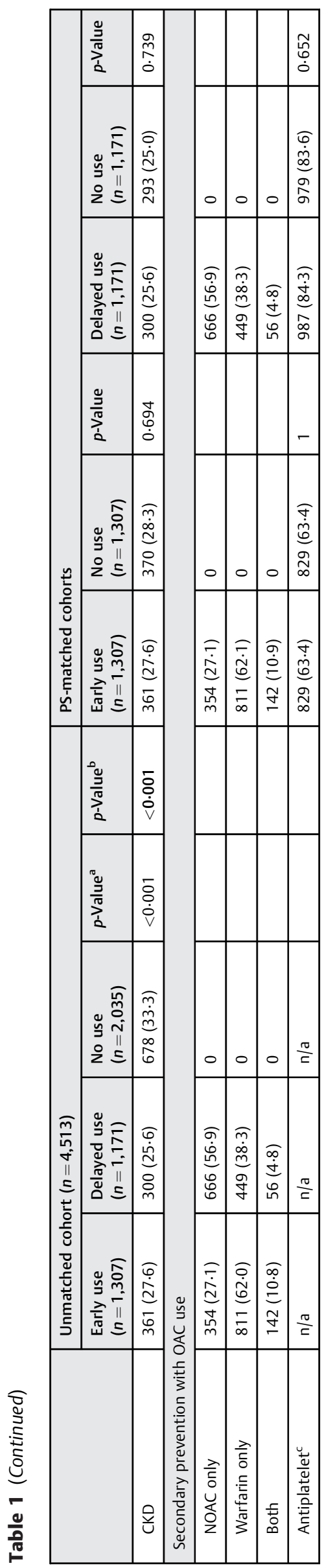

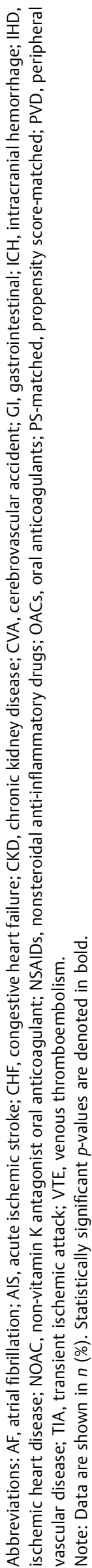

\section{Net Clinical Benefit for OAC Use}

In patients with mild or moderate stroke, early or delayed use of OACs was associated with a statistically significant $\mathrm{NCB}$, as opposed to no OAC use (-Table 4). In patients with severe stroke, use of OACs, compared with no use, was also associated with a NCB although the benefit did not reach statistical significance in the delayed use group.

\section{Discussion}

This study provided the first evidence of a large-scale population and evaluated the effects of early or delayed OAC initiation in a population with AF after AIS by PS-matched cohort on each day since admission, stratified by stroke severity, to overcome immortal-time biases by emulating RCTs. Herein, the key finding was that: first, both early and delayed OAC use could reduce the risk of composite and effectiveness outcomes for each stroke severity. Second, compared with delayed use of OAC, early use, based on the recommendations of current clinical guidelines, was not associated with an excessive risk of composite outcomes. Third, in subjects with severe stroke, early treatment may result in a higher bleeding risk when compared with delayed treatment, despite presenting similar risks for the effectiveness and composite outcomes.

Previous observational studies regarding the timing of OAC initiation after AIS have presented conflicting results. For example, Paciaroni et al have reported that the optimal time to initiate OACs was 4 to 14 days from stroke onset. ${ }^{8}$ Similar results have been observed in the RAF-NOAC study, with the lowest composite rates of recurrence and major bleeding for those who initiated NOACs between 3 and 14 days. ${ }^{9}$ The 2018 American Heart Association/American Stroke Association guidelines recommended that secondary prevention with OACs should be appropriately instituted within the first 2 weeks, ${ }^{38}$ whereas United Kingdom guidelines ${ }^{39}$ recommended that OAC administration be deferred until at least 14 days from the onset in patients with disabling ischemic stroke.

However, more recent studies have not supported the 14day recommendation. Yaghi et al have conducted a registry from eight comprehensive stroke centers and found that OACs started in the 0- to 3-day period were not associated with higher recurrent ischemic events or ICH when compared with those initiated at 4 to 14 days. ${ }^{40}$ Clinical Relevance of Microbleeds In Stroke-2 (CROMIS-2) also suggested that early OAC (0-4 days) after AF-related AIS or TIA was not associated with a difference in the composite outcome of stroke, TIA, or death at 90 days, when compared with delayed OAC ( $\geq 5$ days or never started). ${ }^{41}$

Given that the delayed initiation of OAC was not associated with obvious clinical benefits, early use of OACs in mild and moderate AIS patients with AF might be a reasonable alternative. Our finding is consistent with the previous two small randomized trials, ${ }^{42,43}$ which provided reassurance regarding the safety of early initiation of administration of rivaroxaban or dabigatran in patients with mild-to-moderate ischemic stroke (NIHSS $<9$ ). 


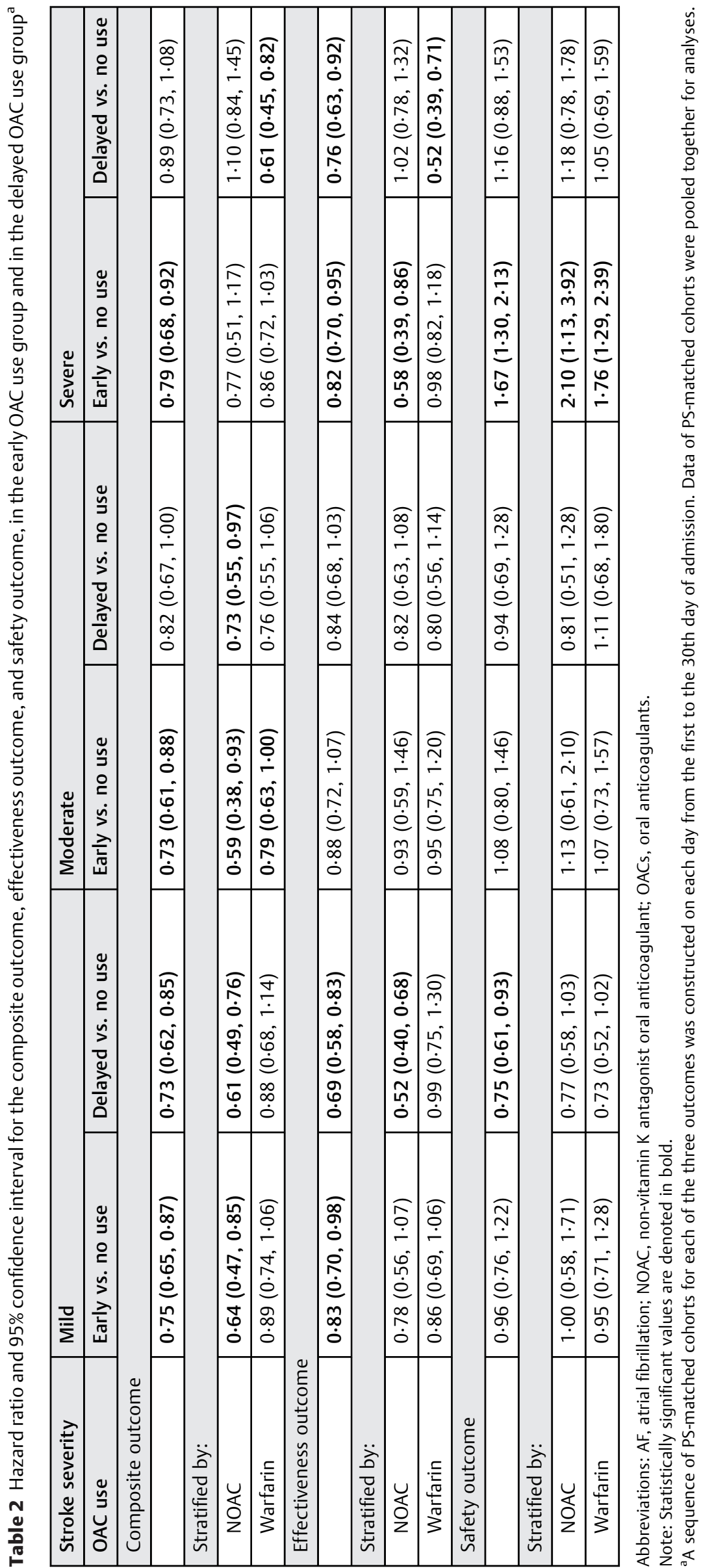


Table 3 Hazard ratio and 95\% confidence intervals for the composite outcome, effectiveness outcome, and safety outcome, comparing the early use with the delayed use of OACs in mixed treatment comparison ${ }^{a}$

\begin{tabular}{|c|c|c|c|}
\hline Stroke severity & Mild & Moderate & Severe \\
\hline Composite outcome & $1.03(0.83,1.27)$ & $0.89(0.68,1.17)$ & $0.89(0.69,1.14)$ \\
\hline \multicolumn{4}{|l|}{ Stratified by: } \\
\hline NOAC & $1.05(0.73,1.52)$ & $0.81(0.48,1.37)$ & $0.70(0.43,1.15)$ \\
\hline Warfarin & $1.01(0.74,1.39)$ & $1.04(0.70,1 \cdot 55)$ & $1.41(0.99,2.00)$ \\
\hline Effectiveness outcome & $1.20(0.94,1.54)$ & $1.05(0.79,1.40)$ & $1.08(0.85,1.38)$ \\
\hline \multicolumn{4}{|l|}{ Stratified by: } \\
\hline NOAC & $1.50(0.99,2.28)$ & $1.13(0.67,1.92)$ & $0.57(0.35,0.91)$ \\
\hline Warfarin & $0.87(0.61,1.23)$ & $1.19(0.78,1.82)$ & $1.88(1.33,2.68)$ \\
\hline Safety outcome & $1.28(0.93,1.76)$ & $1 \cdot 15(0.75,1 \cdot 77)$ & $1.44(0.99,2.09)$ \\
\hline \multicolumn{4}{|l|}{ Stratified by: } \\
\hline NOAC & $1 \cdot 30(0.70,2.40)$ & $1.40(0.65,3.01)$ & $1.78(0.84,3.75)$ \\
\hline Warfarin & $1.30(0.83,2.04)$ & $0.96(0.52,1.79)$ & $1.68(0.998,2.82)$ \\
\hline
\end{tabular}

Abbreviations: AF, atrial fibrillation; NOAC, non-vitamin K antagonist oral anticoagulant; OACs, oral anticoagulants. Note: Statistically significant values are denoted in bold.

aReference group for all mixed treatment comparison was the delayed OAC use.

Previous recommendations for the delayed initiation of OAC were based on concerns of hemorrhagic transformation after AIS. However, in our analysis, at least in subjects with mild-tomoderate stroke severity, a delayed OAC use for secondary prevention in patients with AF and AIS is not an evidence-based recommendation and should not be employed as routine clinical practice; however, in severe patients with AIS, delaying the use of OACs may reduce the risk of bleeding events. RCTs are needed to define the appropriate timing of OACs initiation.

One of the major advantages of our study is the large study population from a nationwide cohort, providing the opportunity to perform PS matching with sufficient event numbers for statistical inference. Another important strength is the comprehensive analytic framework in our study, especially the approach in dealing with immortal-time bias. For research questions involving strategies with different timings, immortal-time bias and confounding bias are difficult issues to resolve in observational studies. By utilizing the day-by-day PS-matching approach, we reduced the immortal-time bias and confounding bias when comparing different strategies. Furthermore, in the present real-world cohorts, we employed the novel mixed treatment comparison meta-analysis techniques for indirect comparisons to obtain relative effects of early versus delayed OAC use in AF patients with AIS.

Several limitations of the present study need to be acknowledged. First, selection bias is an inherent limitation of observational studies. Second, the disease status and outcomes were identified by validated algorithms, ${ }^{16-23,28}$ which might not represent patients' real conditions as the codes were designed to claim health insurance. Third, there may be residual confounding from unmeasured or unknown covariates as NHIRD was unable to provide labora- tory data such as international normalized ratio to evaluate the controlled efficacy of VKAs or imaging data, including CT scan, MRI, and echocardiography, to fully evaluate the clinical status. Claims-based databases also lack the information regarding stroke lesion volume; therefore, the current study applied the validated tool, $\mathrm{SSI},{ }^{25-27}$ for assessing stroke severity rather than NIHSS. The latter, however, is the most widely accepted tool to assess the severity of stroke. Fourth, our study samples were recruited repeatedly in different cohorts to imitate an RCT design. Our estimated results could be confounded by unsatisfied independence. Owing to similar inclusion criteria, methodology, and controlled variables in our study, the consistency assumption of the indirect comparisons is less concerning. ${ }^{44}$ Lastly, the delayed or early initiation of OACs was based on current guideline recommendations, and future studies to determine the most appropriate timing to resume OACs are warranted.

\section{Conclusion}

In patients with AF admitted for AIS, early initiation of OACs and the routine delayed use appeared to result in a comparable risk of composite clinical outcome across the levels of stroke severity. The risk of bleeding events seemed to be similar for all the OAC use groups in patients with mild-tomoderate AIS. However, such a risk was particularly concerning for patients with severe AIS who resumed OACs early. The current study findings support an early OAC initiation in AF patients with mild-to-moderate AIS and a routine delayed use of OACs in those with severe AIS to avoid a serious bleeding event. The optimal timing of OAC initiation after AIS requires further investigation. 


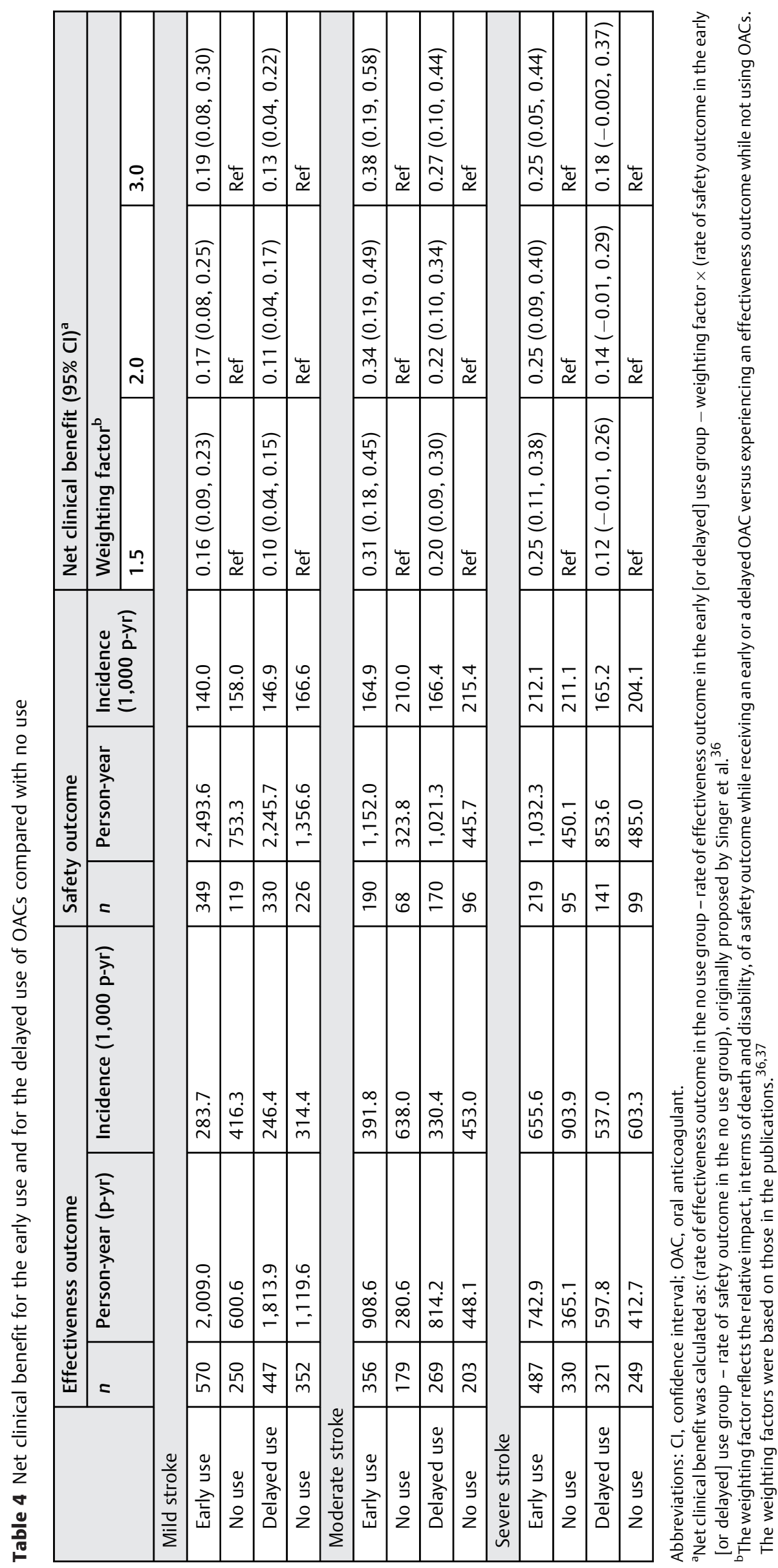




\section{What is known about this topic?}

- Evidence has suggested that high-risk patients with atrial fibrillation (AF) should routinely administer lifelong oral anticoagulants (OACs) for secondary stroke prevention.

- The 2018 European Heart Rhythm Association (EHRA) practical guide proposed a "1-3-6-12 days rule" to resume OAC after an acute ischemic stroke in patients with $\mathrm{AF}$, which is an expert consensus opinion, lacking supporting evidence from large-scale randomized controlled trials or real-world observational studies.

\section{What does this paper add?}

- Both early and delayed OAC uses could reduce composite and effectiveness outcomes for each stroke severity.

- Compared with delayed use of OAC, early use, based on the recommendations of current clinical guidelines, was not associated with an excessive risk of composite outcomes.

- In subjects with severe stroke, early treatment may result in a higher bleeding risk when compared with delayed treatment, despite presenting similar risks for the effectiveness and composite outcomes.

\section{Ethical Approval}

The study protocol was approved by ethic committee of Taipei Veterans General Hospital.

\section{Note}

All authors confirm that they had full access to all the data in the study and accept responsibility to submit for publication. However, our study does not contain data from any individual. The datasets used and analyzed during the current study are available from the corresponding author on reasonable request. The authors declare that they have no competing interests. This article reflects the views of the authors and does not represent the U.S. Food and Drug Administration's views or policies.

\section{Author Contributions}

C.-E.C., H.-M.C., and Y.-W.T. conceived and designed the research. Statistical analysis was performed by W.-L. Wu, P.-Y.C., W.-T.W., and H.-C.C.. W.-T.W., P.-Y.C., Y.-W.T., S.-H. C., and H.-M.C. drafted the article. C.-H.C. and C.-E.C. made critical revision of the article for key intellectual content. Each author contributed important intellectual content during article drafting or revision and accepts accountability for the overall work by ensuring that questions pertaining to the accuracy or integrity of any portion of the work are appropriately investigated and resolved. H.-M.C., C.-H.C., C.-E.C., and Y.-W.T. undertake that this study has been reported honestly, accurately, and trans- parently, that no important aspects of the study have been omitted, and that any discrepancies from the study as planned (and, if relevant, registered) have been explained.

\section{Funding}

None.

\section{Conflict of Interest}

G.Y.H.L.: investigator for the OPTIMAS trial; consultant for Bayer/Janssen, BMS/Pfizer, Boehringer Ingelheim, Verseon, and Daiichi-Sankyo; speaker for BMS/Pfizer, Boehringer Ingelheim, and Daiichi-Sankyo. No fees were directly received in person.

\section{References}

1 GBD 2016 Stroke Collaborators. Global, regional, and national burden of stroke, 1990-2016: a systematic analysis for the Global Burden of Disease Study 2016. Lancet Neurol 2019;18(05): 439-458

2 Wolf PA, Abbott RD, Kannel WB. Atrial fibrillation as an independent risk factor for stroke: the Framingham Study. Stroke 1991;22 (08):983-988

3 January CT, Wann LS, Calkins H, et al. 2019 AHA/ACC/HRS focused update of the 2014 AHA/ACC/HRS Guideline for the Management of Patients With Atrial Fibrillation: a report of the American College of Cardiology/American Heart Association Task Force on Clinical Practice Guidelines and the Heart Rhythm Society in collaboration with the Society of Thoracic Surgeons. Circulation 2019;140(02):e125-e151

4 Steffel J, Verhamme P, Potpara TS, et al; ESC Scientific Document Group. The 2018 European Heart Rhythm Association Practical Guide on the use of non-vitamin K antagonist oral anticoagulants in patients with atrial fibrillation. Eur Heart J 2018;39(16): 1330-1393

5 Paciaroni M, Bandini F, Agnelli G, et al. Hemorrhagic transformation in patients with acute ischemic stroke and atrial fibrillation: time to initiation of oral anticoagulant therapy and outcomes. J Am Heart Assoc 2018;7(22):e010133

6 Kirchhof P, Benussi S, Kotecha D, et al; ESC Scientific Document Group. 2016 ESC guidelines for the management of atrial fibrillation developed in collaboration with EACTS. Eur Heart J 2016;37 (38):2893-2962

7 Wilson D, Ambler G, Shakeshaft C, et al; CROMIS-2 collaborators. Cerebral microbleeds and intracranial haemorrhage risk in patients anticoagulated for atrial fibrillation after acute ischaemic stroke or transient ischaemic attack (CROMIS-2): a multicentre observational cohort study. Lancet Neurol 2018;17(06):539-547

8 Paciaroni M, Agnelli G, Falocci N, et al. Early recurrence and cerebral bleeding in patients with acute ischemic stroke and atrial fibrillation: effect of anticoagulation and its timing: the RAF study. Stroke 2015;46(08):2175-2182

9 Paciaroni M, Agnelli G, Falocci N, et al. Early recurrence and major bleeding in patients with acute ischemic stroke and atrial fibrillation treated with non-vitamin-K oral anticoagulants (RAFNOACs) study. J Am Heart Assoc 2017;6(12):e007034

10 Yoshimura S, Koga M, Sato S, et al; SAMURAI Study Investigators. Two-year outcomes of anticoagulation for acute ischemic stroke with nonvalvular atrial fibrillation - SAMURAI-NVAF study. Circ J 2018;82(07):1935-1942

11 Seiffge DJ, Traenka C, Polymeris A, et al. Early start of DOAC after ischemic stroke: risk of intracranial hemorrhage and recurrent events. Neurology 2016;87(18):1856-1862

12 Macha K, Volbers B, Bobinger T, et al. Early initiation of anticoagulation with direct oral anticoagulants in patients after 
transient ischemic attack or ischemic stroke. J Stroke Cerebrovasc Dis 2016;25(09):2317-2321

13 Cappellari M, Carletti M, Danese A, Bovi P. Early introduction of direct oral anticoagulants in cardioembolic stroke patients with non-valvular atrial fibrillation. J Thromb Thrombolysis 2016;42 (03):393-398

14 Seiffge DJ, Paciaroni M, Wilson D, et al; CROMIS-2, RAF, RAFDOAC, SAMURAI, NOACISP LONGTERM, Erlangen and Verona registry collaborators. Direct oral anticoagulants versus vitamin $\mathrm{K}$ antagonists after recent ischemic stroke in patients with atrial fibrillation. Ann Neurol 2019;85(06):823-834

15 Mizoguchi T, Tanaka K, Toyoda K, et al; SAMURAI Study Investigators. Early initiation of direct oral anticoagulants after onset of stroke and short- and long-term outcomes of patients with nonvalvular atrial fibrillation. Stroke 2020;51(03):883-891

16 Chan Y-H, Yen K-C, See L-C, et al. Cardiovascular, bleeding, and mortality risks of dabigatran in asians with nonvalvular atrial fibrillation. Stroke 2016;47(02):441-449

17 Chao TF, Liu CJ, Wang KL, et al. Using the CHA2DS2-VASc score for refining stroke risk stratification in 'low-risk' Asian patients with atrial fibrillation. J Am Coll Cardiol 2014;64(16):1658-1665

18 Chao TF, Liu CJ, Wang KL, et al. Should atrial fibrillation patients with 1 additional risk factor of the CHA2DS2-VASc score (beyond sex) receive oral anticoagulation? J Am Coll Cardiol 2015;65(07): 635-642

19 Chao TF, Wang KL, Liu CJ, et al. Age threshold for increased stroke risk among patients with atrial fibrillation: a nationwide cohort study from Taiwan. J Am Coll Cardiol 2015;66(12):1339-1347

20 Chao T-F, Liu C-J, Tuan T-C, et al. Rate-control treatment and mortality in atrial fibrillation. Circulation 2015;132(17):1604-1612

21 Cheng CL, Kao YH, Lin SJ, Lee CH, Lai ML. Validation of the National Health Insurance Research Database with ischemic stroke cases in Taiwan. Pharmacoepidemiol Drug Saf 2011;20(03):236-242

22 Hsieh CY, Chen CH, Li CY, Lai ML. Validating the diagnosis of acute ischemic stroke in a National Health Insurance claims database. J Formos Med Assoc 2015;114(03):254-259

23 Lin LJ, Cheng MH, Lee CH, Wung DC, Cheng CL, Kao Yang YH. Compliance with antithrombotic prescribing guidelines for patients with atrial fibrillation-a nationwide descriptive study in Taiwan. Clin Ther 2008;30(09):1726-1736

24 Chang C-H, Lee Y-C, Tsai C-T, et al. Continuation of statin therapy and a decreased risk of atrial fibrillation/flutter in patients with and without chronic kidney disease. Atherosclerosis 2014;232 (01):224-230

25 Sung SF, Hsieh CY, Lin HJ, et al. Validity of a stroke severity index for administrative claims data research: a retrospective cohort study. BMC Health Serv Res 2016;16(01):509

26 Sung S-F, Chen SC-C, Hsieh C-Y, Li C-Y, Lai EC-C, Hu Y-H. A comparison of stroke severity proxy measures for claims data research: a population-based cohort study. Pharmacoepidemiol Drug Saf 2016;25(04):438-443

27 Sung S-F, Hsieh C-Y, Kao Yang Y-H, et al. Developing a stroke severity index based on administrative data was feasible using data mining techniques. J Clin Epidemiol 2015;68(11):1292-1300

28 Lin CC, Hu HY, Luo JC, et al. Risk factors of gastrointestinal bleeding in clopidogrel users: a nationwide population-based study. Aliment Pharmacol Ther 2013;38(09):1119-1128

29 Suissa S. Immortal time bias in pharmaco-epidemiology. Am J Epidemiol 2008;167(04):492-499
30 Hernán MA, Sauer BC, Hernández-Díaz S, Platt R, Shrier I. Specifying a target trial prevents immortal time bias and other selfinflicted injuries in observational analyses. J Clin Epidemiol 2016; 79:70-75

31 Rosenbaum PR, Rubin DB. Constructing a control group using multivariate matched sampling methods that incorporate the propensity score. Am Stat 1985;39(01):33-38

32 Hernán MA, Alonso A, Logan R, et al. Observational studies analyzed like randomized experiments: an application to postmenopausal hormone therapy and coronary heart disease. Epidemiology 2008;19(06):766-779

33 Fine JP, Gray RJ. A proportional hazards model for the subdistribution of a competing risk. J Am Stat Assoc 1999;94(446): 496-509

34 Austin PC, Lee DS, Fine JP. Introduction to the analysis of survival data in the presence of competing risks. Circulation 2016;133 (06):601-609

35 Hoyer A. Metaanalysis with R. G.Schwarzer, J. R.Carpenter, G. Rücker (2015). Berlin, DE: Springer. ISBN: 978-3-319-21415-3. Biom J 2017;59(01):216-217

36 Singer DE, Chang Y, Fang MC, Borowsky LH, Pomernacki NK, Udaltsova $\mathrm{N}$, et al. The net clinical benefit of warfarin anticoagulation in atrial fibrillation. Ann Intern Med 2009;151:297-305

37 Chao TF, Lip GY, Liu CJ, et al. Validation of a modified CHA2DS2VASc score for stroke risk stratification in asian patients with atrial fibrillation: a nationwide cohort study. Stroke 2016;47(10): 2462-2469

38 Powers WJ, Rabinstein AA, Ackerson T, et al. Guidelines for the early management of patients with acute ischemic stroke: 2019 update to the 2018 guidelines for the early management of acute ischemic stroke: a guideline for healthcare professionals from the American Heart Association/American Stroke Association. Stroke 2019;50(12):e344-e418

39 Rudd AG, Bowen A, Young GR, James MA. The latest national clinical guideline for stroke. Clin Med (Lond) 2017;17(02): 154-155

40 Yaghi S, Trivedi T, Giles J, et al. Abstract 119: initiating oral anticoagulation 4 to 14 days after a cardioembolic stroke is not associated with a reduction in ischemic or hemorrhagic events: the IAC Multicenter cohort. Stroke 2020;51(Suppl 1):A119-A119

41 Wilson D, Ambler G, Banerjee G, et al; Clinical relevance of Microbleeds in Stroke (CROMIS-2) collaborators. Early versus late anticoagulation for ischaemic stroke associated with atrial fibrillation: multicentre cohort study. J Neurol Neurosurg Psychiatry 2019;90(03):320-325

42 Hong K-S, Kwon SU, Lee SH, et al; Phase 2 Exploratory Clinical Study to Assess the Effects of Xarelto (Rivaroxaban) Versus Warfarin on Ischemia, Bleeding, and Hospital Stay in Acute Cerebral Infarction Patients With Non-valvular Atrial Fibrillation (Triple AXEL) Study Group. Rivaroxaban vs warfarin sodium in the ultra-early period after atrial fibrillation-related mild ischemic stroke: a randomized clinical trial. JAMA Neurol 2017;74(10): 1206-1215

$43 \mathrm{Ng} \mathrm{KH}$, Sharma M, Benavente O, et al. Dabigatran following acute transient ischemic attack and minor stroke II (DATAS II). Int J Stroke 2017;12(18):910-914

44 Shim S, Yoon B-H, Shin I-S, Bae J-M. Network meta-analysis: application and practice using Stata. Epidemiol Health 2017;39: e2017047-e2017047 